\title{
Two Axiomatic Approaches to Decision Making Using Possibility Theory*
}

\author{
Phan H. Giang ${ }^{\dagger}$ \\ Computer Aided Diagnosis \& Therapy Group \\ Siemens Medical Solutions H32 \\ 51 Valley Stream Pkwy \\ Malvern, PA 19355 USA \\ Phone: 610-448-4324, Fax: 610-448-4274 \\ phan.giang@siemens.com \\ Prakash P. Shenoy \\ University of Kansas, School of Business \\ 1300 Sunnyside Avenue, Summerfield Hall \\ Lawrence, KS 66045-7585, USA \\ pshenoy@ku.edu
}

September 22, 2003

\begin{abstract}
This paper proposes a utility theory for decision making under uncertainty that is described by possibility theory. We show that our approach is a natural generalization of the two axiomatic systems that correspond to pessimistic and optimistic decision criteria proposed by Dubois et al. The generalization is achieved by removing axioms that are supposed to reflect attitudes toward uncertainty, namely, pessimism and optimism. In their place we adopt an axiom that imposes an order on a class of canonical lotteries that realize either in the best or in the worst prize. We prove an expected utility theorem for the generalized axiomatic system based on the newly introduced concept of binary utility.
\end{abstract}

Keywords: Decision theory; Uncertainty; Possibility theory; Utility theory

\footnotetext{
${ }^{*}$ A preliminary version of this paper appeared in the proceedings of 17 th Conference on Uncertainty in Artificial Intelligence [10]. The authors are grateful to the referees for their insightful comments and constructive suggestions.

${ }^{\dagger}$ Corresponding author. This work has been done at the University of Kansas.
} 


\section{Introduction}

The goal of this paper is twofold. First, we propose a utility theory for decision making under uncertainty that is described by the possibility theory. Second, we show that our approach generalizes a recent proposal by Dubois et al. [4].

In recent years, there has been a growing interest in qualitative decision making within the artificial intelligence and decision analysis communities [2]. The aim of the research is to deal with situations where probability and utility inputs required by the classical Bayesian decision theory are difficult to assess or when the postulates underlying this theory are challenged. Among several alternative approaches for modeling uncertainty, belief function theory [15, 16], interval-valued probability [12, 19], Spohn's theory of epistemic belief [17] and possibility theory $[20,6]$ have been extensively studied.

Once uncertainty has been represented, the next step is to determine how it can be used for making decisions. For belief functions and interval-valued probabilities, where a situation is represented by a family of probability distributions instead of a single one, the classical Bayesian decision theory can be used in two ways. Either, a probability distribution from the family is selected according to some criteria (e.g., pignistic probability [16]), then, for each alternative an expected utility can be calculated. Or, for each alternative, a range of expectations is assessed with respect to probability functions in the family [19]. Comparison between alternatives boils down to comparing lower and upper expectations. Several scholars argue for the use of Choquet expected utility as an extension of von Neumann - Morgenstern classical expected utility in situations where uncertainty is represented by non-additive probability $[14,13]$. For possibility theory, which apparently has no such strong connection with probability, the technique is of little use. In recent years, efforts have been made by Dubois, Fargier, Godo, Prade, Sabbadin, and Zapico to create an axiomatic basis for possibility-based decision theory. The efforts are in two different but related directions following von Neumann - Morgenstern and Savage [4, 7]. Also, we have proposed a different axiomatic system for decision making for possibility-based decision theory [10]. In this paper, we compare these two proposals.

An outline of this article is as follows. In section 2, the proposal by Dubois et al. for a decision theory with possibility theory is reviewed. In section 3 , we present a new system of axioms. The concept of binary utility is introduced. The representation theorem based on binary utility for the new axiom system is proved. In section 4, we compare our approach against one argued by Dubois and others. We prove a theorem stating that the two axiomatic systems by Dubois et al. are special cases of our system. Examples that illustrate the calculations with different utility functions are provided. Finally, in section 5, we conclude with some remarks. 


\section{Pessimistic and Optimistic Utilities}

In this section, we review with some modification of terminology, the proposal that has been described in [4]. A decision maker faces a situation that can be described as follows. There is a set $S$ of possible states and a finite uncertainty scale $V$. Without loss of generality, $V$ is assumed to be a set of points in the closed unit interval $[0,1]$. Order $\geq$ on $V$ is defined in the usual way. Uncertainty about which possible state will occur is represented by a possibility distribution that is a mapping $\pi: S \rightarrow V$ such that $\max _{s \in S} \pi(s)=1$. The possibility of a subset $A \subseteq S, \pi(A) \stackrel{\text { def }}{=} \max _{s \in A} \pi(s)$. Let $X=\left\{x_{1}, x_{2}, \ldots x_{n}\right\}$ denote a finite set of consequences or prizes. To avoid trivialities, $X$ is assumed to have at least two distinct elements, i.e., $n \geq 2$.

A decision is a mapping $d: S \rightarrow X$. That is, decision $d$ results in consequence $d(s)$ if state $s$ occurs. Notice that each decision $d$ induces a possibility distribution $\pi_{d}$ on the set $X$ of consequences in the following sense: $\pi_{d}(x) \stackrel{\text { def }}{=} \pi\left(d^{-1}(x)\right)$ where $d^{-1}(x)=\{s \in S \mid d(s)=x\}$. A possibility distribution on $X$ is also called a possibilistic lottery. Later on in this paper we just call it lottery. Sometimes, we use a list notation $\left[\pi\left(x_{1}\right) / x_{1}, \pi\left(x_{2}\right) / x_{2} \ldots \pi\left(x_{n}\right) / x_{n}\right]$ to denote a lottery $\pi$ with the convention that impossible consequences (consequences $x$ with $\pi(x)=0$ ) are omitted. The set of lotteries is denoted by $\Pi_{X}$. Notice that $\Pi_{X}$ is closed under the operation possibilistic mix defined as follows. A possibilistic mixture of $m$ possibility functions $\pi_{1}, \ldots \pi_{m}$ with possibilities $\lambda_{1}, \ldots \lambda_{m}$ such that $\max _{i \in\{1,2, \ldots m\}}\left\{\lambda_{i}\right\}=1$ is defined as

$$
\left[\lambda_{1} / \pi_{1}, \ldots \lambda_{m} / \pi_{m}\right](x) \stackrel{\text { def }}{=} \max _{i \in\{1,2, \ldots m\}} \min \left(\lambda_{i}, \pi_{i}(x)\right) .
$$

See [3] for more details.

To the decision maker, the value of decision $d$ is the same as the value of the induced lottery $\pi_{d}$. And thanks to the mixture construct, the set $\Pi_{X}$ is rich enough to encode not only a simple lottery but also a "compound" lottery. Intuitively, the concept of a possibilistic mixture is similar to that of multi-stage probabilistic lottery. Notice that each prize $x \in X$ can be viewed as a lottery denoted by $\pi_{x}$ where $\pi_{x}(x)=1$ and $\pi_{x}\left(x^{\prime}\right)=0$ for $x^{\prime} \neq x$. Later on, symbols $x$ and $\pi_{x}$ will be used interchangeably. Thus, a preference for decisions is the same as a preference for lotteries. Let $\succeq$ (read as "at least as good as") denote a preference relation on the set $\Pi_{X}$ of possibility distributions. (Two derivative relations $\succ$ for strict preference, and $\sim$ for indifference are also used.)

Since $X \subseteq \Pi_{X}, \succeq$ applies to $X$ as well. Since $X$ is finite and has at least two distinct elements, we can single out a best prize and a worst prize denoted by $\bar{x}$ and $\underline{x}$ respectively i.e., $\bar{x} \succeq x$ and $x \succeq \underline{x} \forall x \in X$. These two elements are called the anchors of set $X$.

A preference relation can be characterized by a system of axioms it must satisfy. Or it can be represented by a utility function that maps $\Pi_{X}$ into some (finite) linearly ordered scale $U$ called the utility scale. We will assume $\sup (U)=$ 1 and $\inf (U)=0$. The preference relation $\geq$ is used for both numerical and utility comparison. 
Dubois et al. propose two types of utilities called pessimistic and optimistic utilities. In [4], the authors study two axiomatic systems in the style of von Neumann and Morgenstern. They show that a preference relation satisfying one of the axiom systems is represented by the pessimistic utility function, and the other system is represented by the optimistic utility function. In [7], those two utility functions are justified by axiom systems in the style of Savage. The difference is that in the former approach, a possibility function on states is assumed to be given, whereas in the latter approach such a function is deduced from a preference relation on the set of actions.

\subsection{Pessimistic Utility}

The pessimistic utility concept needs the following ingredients: a function

$$
u: X \rightarrow U
$$

that is called utility assignment of prizes such that $u(\bar{x})=1$ and $u(\underline{x})=0$; a function

$$
n: U \rightarrow U
$$

that is an order reversing involution in $U$ i.e. $n(1)=0, n(0)=1$ and $n\left(u_{1}\right) \geq n\left(u_{2}\right)$ whenever $u_{2} \geq u_{1}$; and a function

$$
h: V \rightarrow U
$$

that is an order preserving mapping from uncertainty scale $V$ onto utility scale $U$ such that $h(1)=1$ and $h(0)=0$. A pessimistic qualitative utility function $Q U^{-}: \Pi_{X} \rightarrow U$ is defined as follows:

$$
Q U^{-}(\pi) \stackrel{\text { def }}{=} \min _{x \in X} \max (n h(\pi(x)), u(x))
$$

where $n h \stackrel{\text { def }}{=} n \circ h$ is composition of $n$ and $h$. We list a simple lemma whose proof is straightforward

\section{Lemma 1}

$Q U^{-}(\underline{x})=Q U^{-}([1 / \bar{x}, 1 / \underline{x}])=0$

$Q U^{-}(\bar{x})=1$

$Q U^{-}([1 / \bar{x}, \lambda / \underline{x}])=n h(\lambda)$.

Given a utility function $u: \Pi_{X} \rightarrow U$, one can define a preference relation $\succeq_{u}$ on $\Pi_{X}$ as $\pi_{1} \succeq_{u} \pi_{2}$ iff $u\left(\pi_{1}\right) \geq u\left(\pi_{2}\right)$. We say $\succeq_{u}$ is represented by $u$. A preference relation can be characterized by its properties stated as axioms. The authors study the following axiom system consisting of four axioms denoted by $\mathcal{S}_{P}$.

$A 1^{-}$(Total pre-order) $\succeq$ is reflexive, transitive and complete.

$A 2^{-}$(Uncertainty aversion) If $\pi^{\prime} \geq \pi$ then $\pi \succeq \pi^{\prime}$.

$A 3^{-}$(Substitutability) If $\pi_{1} \sim \pi_{2}$ then $\left[\lambda / \pi_{1}, \mu / \pi\right] \sim\left[\lambda / \pi_{2}, \mu / \pi\right]$. 
$A 4^{-}$(Continuity) $\forall \pi \in \Pi_{X}, \exists \lambda \in V \quad \pi \sim[1 / \bar{x}, \lambda / \underline{x}]$.

Dubois et al. prove the following representation theorem.

Theorem 1 A preference relation $\succeq$ on $\Pi_{X}$ satisfies system $\mathcal{S}_{P}$ iff there exist functions $u, n, h$ and pessimistic utility function $Q U^{-}$defined by equations (2, 3, 4, 5) such that $\pi \succeq \pi^{\prime}$ iff $Q U^{-}(\pi) \geq Q U^{-}\left(\pi^{\prime}\right)$.

\subsection{Optimistic Utility}

The authors also consider another utility function that supposedly captures the optimistic behavior of decision makers. The optimistic qualitative utility function $Q U^{+}: \Pi_{X} \rightarrow U$ is defined as follows

$$
Q U^{+}(\pi) \stackrel{\text { def }}{=} \max _{x \in X} \min (h(\pi(x)), u(x))
$$

\section{Lemma 2}

$Q U^{+}(\underline{x})=0$

$Q U^{+}(\bar{x})=Q U^{+}([1 / \bar{x}, 1 / \underline{x}])=1$

$Q U^{+}([\lambda / \bar{x}, 1 / \underline{x}])=h(\lambda)$.

The system $\mathcal{S}_{O}$ of axioms that characterizes $Q U^{+}$is obtained from $\mathcal{S}_{P}$ by substituting axioms $A 2^{-}$and $A 4^{-}$by their diametrical counterparts $A 2^{+}$and $A 4^{+}$, respectively, where

$$
\begin{aligned}
& A 2^{+} \text {(Uncertainty attraction) If } \pi \geq \pi^{\prime} \text { then } \pi \succeq \pi^{\prime} . \\
& A 4^{+} \text {(Continuity) } \forall \pi \in \Pi_{X}, \exists \lambda \in V \quad \pi \sim[\lambda / \bar{x}, 1 / \underline{x}] .
\end{aligned}
$$

They also prove a representation theorem, similar to Theorem 1, for $Q U^{+}$ and $\mathcal{S}_{O}$. Note that decision based on $Q U^{-}, Q U^{+}$could be viewed as extensions of the minimax and maximax rules (for details about these rules see [1]).

We end this review section with some numerical examples illustrating the use of pessimistic and optimistic utilities.

Example 1. Let $X=\left\{\bar{x}, x_{2}, x_{3}, \underline{x}\right\} . \bar{x} \succ x_{2} \succ x_{3} \succ \underline{x} . V=\{1, .7, .5,0\}$ and $U=\{1, .5, .3,0\}$. Suppose that the decision maker is considering three lotteries $\pi_{1}, \pi_{2}, \pi_{3} \in \Pi_{X}$

\begin{tabular}{rrrrr}
$X$ & $\bar{x}$ & $x_{2}$ & $x_{3}$ & $\underline{x}$ \\
\hline$\pi_{1}$ & .7 & 1.0 & .5 & .5 \\
$\pi_{2}$ & .5 & .7 & 0 & 1.0 \\
$\pi_{3}$ & 1.0 & 1.0 & 1.0 & 1.0
\end{tabular}

Since the cardinality of $X$ equals that of utility scale $U$ the only consistent utility assignment is

$$
\begin{array}{lllll}
X & \bar{x} & x_{2} & x_{3} & \underline{x} \\
\hline u & 1 & .5 & .3 & 0
\end{array}
$$


In order to decide on a preference relation between the lotteries, one needs to elicit the utility by revealing the indifference between the prizes and canonical lotteries, which end up either with the best or the worst prize. Because the cardinality of $X$ equals that of uncertainty scale $V$, for each of axioms $A 4^{-}$and $A 4^{+}$, there is only one set of indifference that satisfies it.

Case 1. For $A 4^{-}$, the set of indifference is $\bar{x} \sim[1 / \bar{x}, 0 / \underline{x}], x_{2} \sim[1 / \bar{x}, .5 / \underline{x}]$, $x_{3} \sim[1 / \bar{x}, .7 / \underline{x}]$ and $\underline{x} \sim[1 / \bar{x}, 1 / \underline{x}]$. This decision maker is classified as pessimistic. By lemma 1, function $n h$ that maps $V$ to $U$ must be $n h(1)=0$, $n h(.7)=.3, n h(.5)=.5$ and $n h(0)=1$. This function could be a composition of $n$ and $h$ given as follows.

\begin{tabular}{|c|c|c|c|c|c|c|}
\hline$U$ & $\begin{array}{ll}1 & .5\end{array}$ & .3 & 0 & $V$ & 1 & 5 \\
\hline & & & & $\bar{h}$ & & \\
\hline
\end{tabular}

Using definition (5), we calculate utility for $\pi_{1}, \pi_{2}$ and $\pi_{3}$

$$
\begin{aligned}
Q U^{-}\left(\pi_{1}\right) & =\min \left\{\begin{array}{l}
\max (n h(.7), u(\bar{x})) \\
\max \left(n h(1), u\left(x_{2}\right)\right) \\
\max \left(n h(.5), u\left(x_{3}\right)\right) \\
\max (n h(.5), u(\underline{x}))
\end{array}\right\}=\min \left\{\begin{array}{c}
\max (.3,1) \\
\max (0, .5) \\
\max (.5, .3) \\
\max (.5,0)
\end{array}\right\} \\
& =\min \{1, .5, .5, .5\}=.5 .
\end{aligned}
$$

And similarly for $\pi_{2}, \pi_{3}$ we have $Q U^{-}\left(\pi_{2}\right)=0$ and $Q U^{-}\left(\pi_{3}\right)=0$. Thus, $Q U^{-}$ (for the decision maker) ranks $\pi_{1} \succ \pi_{2} \sim \pi_{3}$.

Case 2. For optimistic $A 4^{+}$, the set of indifference is $\bar{x} \sim[1 / \bar{x}, 1 / \underline{x}]$, $x_{2} \sim[.7 / \bar{x}, 1 / \underline{x}], x_{3} \sim[.5 / \bar{x}, 1 / \underline{x}]$ and $\underline{x} \sim[0 / \bar{x}, 1 / \underline{x}]$. By lemma 2 , we know that function $h$ must be the same as above.

$$
\begin{aligned}
Q U^{+}\left(\pi_{1}\right) & =\max \left\{\begin{array}{c}
\min (h(.7), u(\bar{x})) \\
\min \left(h(1), u\left(x_{2}\right)\right) \\
\min \left(h(.5), u\left(x_{3}\right)\right) \\
\min (h(.5), u(\underline{x}))
\end{array}\right\}=\max \left\{\begin{array}{c}
\min (.5,1) \\
\min (1, .5) \\
\min (.3, .3) \\
\min (.3,0)
\end{array}\right\} \\
& =\max \{.5, .5, .3,0\}=.5 .
\end{aligned}
$$

And similarly for $\pi_{2}, \pi_{3}$ we have $Q U^{+}\left(\pi_{2}\right)=.5$ and $Q U^{+}\left(\pi_{3}\right)=1$. Thus, $Q U^{+}$ (for the optimistic decision maker) ranks $\pi_{3} \succ \pi_{1} \sim \pi_{2}$.

\section{Binary Possibilistic Utility}

In this section, we investigate a new system of axioms for preference relations on the set of lotteries. This system is derived from our previous proposal [9] for qualitative decision making tailored for Spohn's theory of epistemic beliefs [17]. The above example demonstrates the important role played by the lotteries that realize either in the best prize $\bar{x}$ or the worst prize $\underline{x}$. We will use the term canonical for them. The set of all canonical lotteries is denoted by $\mathcal{C}$

$$
\mathcal{C} \stackrel{\text { def }}{=}\{[\lambda / \bar{x}, \mu / \underline{x}] \mid \lambda, \mu \in V, \max (\lambda, \mu)=1\} .
$$


We propose the following system of four axioms, denoted by $\mathcal{S}$ (without subscript), for preference relation on lotteries.

$B 1$ (Total pre-order) $\succeq$ is reflexive, transitive and complete.

$B 2$ (Qualitative monotonicity) $\succeq$ restricted to $\mathcal{C}$ satisfies the following condition

$$
[\lambda / \bar{x}, \mu / \underline{x}] \succeq\left[\lambda^{\prime} / \bar{x}, \mu^{\prime} / \underline{x}\right] \quad \text { if } \quad\left\{\begin{array}{l}
\left(1 \geq \lambda \geq \lambda^{\prime} \& \mu=\mu^{\prime}=1\right) \vee \\
\left(\lambda=1 \& \lambda^{\prime}<1\right) \vee \\
\left(\lambda=\lambda^{\prime}=1 \& \mu^{\prime} \geq \mu\right)
\end{array}\right.
$$

$B 3$ (Substitutability) If $\pi_{1} \sim \pi_{2}$ then $\left[\lambda / \pi_{1}, \mu / \pi\right] \sim\left[\lambda / \pi_{2}, \mu / \pi\right]$.

$B 4$ (Continuity) $\forall x \in X, \exists \sigma \in \mathcal{C} \quad x \sim \sigma$.

We list the axioms of $\mathcal{S}$ in the same order as those of $\mathcal{S}_{P}$. Comparing $\mathcal{S}$ with $\mathcal{S}_{P}$, we see immediately that axioms $B 1, B 3$ are the same as $A 1^{-}, A 3^{-}$and $B 4$ is slightly more general than $A 4^{-}$. The crucial difference between two systems is in the contrast between $A 2^{-}$(uncertainty aversion) vs $B 2$ (monotonicity). This similarity tells us that axioms $B 1, B 3$ and $B 4$ could be justified in the same manner as $A 1^{-}, A 3^{-}$and $A 4^{-}$. We will go in more details in section 4 . Now we investigate the properties of $\mathcal{S}$. First, we state a lemma that describes the structure of $\Pi_{X}$.

Lemma 3 Assume $\succeq$ satisfies $\mathcal{S}$ (axioms B1 through B4). For each $\pi \in \Pi_{X}$, there exists a $\sigma \in \mathcal{C}$ such that $\pi \sim \sigma$.

Proof: By definition of possibilistic mixture (1), $\pi$ can be rewritten in the form of a mixture $\left[\pi\left(x_{1}\right) / x_{1}, \pi\left(x_{2}\right) / x_{2}, \ldots \pi\left(x_{n}\right) / x_{n}\right]$. By $B 4$, we have $x_{i} \sim \sigma_{i}$ for $i \in\{1,2, \ldots n\}$ where $\sigma_{i}$ is a canonical lottery $\sigma_{i}=\left[\lambda_{i} / \bar{x}, \mu_{i} / \underline{x}\right]$. By $B 3$, we have $\pi \sim\left[\pi\left(x_{1}\right) / \sigma_{1}, \pi\left(x_{2}\right) / \sigma_{2}, \ldots \pi\left(x_{n}\right) / \sigma_{n}\right]$. Again using the definition of mixture, we have $\left[\pi\left(x_{1}\right) / \sigma_{1}, \pi\left(x_{2}\right) / \sigma_{2}, \ldots \pi\left(x_{n}\right) / \sigma_{n}\right]=[\lambda / \bar{x}, \mu / \underline{x}]$ where

$$
\begin{aligned}
& \lambda=\max _{i \in\{1,2, \ldots n\}} \min \left(\pi\left(x_{i}\right), \lambda_{i}\right) \\
& \mu=\max _{i \in\{1,2, \ldots n\}} \min \left(\pi\left(x_{i}\right), \mu_{i}\right)
\end{aligned}
$$

So $\pi \sim[\lambda / \bar{x}, \mu / \underline{x}]$.

This lemma establishes a homomorphism between the set of lotteries and one of its subset, $\mathcal{C}$, with respect to $\succeq$. It allows us to focus on the set of canonical lotteries, which have a simple structure. Before we can describe a representation theorem for system $\mathcal{S}$, we need an appropriate utility construct. Any ordered set can serve that purpose. A real interval is often used as the utility scale. However, it is well known that in certain situations, it is not convenient to take an one-dimension set for the utility scale. Fishburn [8] shows that if the Archimedian condition in von Neumann - Morgenstern theory is relaxed, lotteries are represented in a lexicographic utility scale, which is a set of tuples of reals equipped with a lexicographic order. 
In the probabilistic case, a canonical lottery can be represented by a single number - the probability $p$ of getting the best prize - because the probability of getting the worst prize is $1-p$. The situation is different, however, in the possibilistic case. The knowledge that the best prize is fully possible $(\lambda=1)$ could imply nothing about the possibility of getting the worst prize ( $\mu$ can take any value in $[0,1]])$. Therefore, to represent a possibilistic canonical lottery, we need two parameters. We propose a concept of the binary utility scale formed from a linearly ordered set. Suppose $W$ is a linearly ordered scale, i.e., a set equipped with an order relation $\geq$. Also, we assume that supremum and infimum of the elements of $\mathrm{W}$ exist and are denoted by 1 and 0 , respectively. We define a set $U_{W}$ of pairs as

$$
U_{W} \stackrel{\text { def }}{=}\{\langle a, b\rangle \mid a, b \in W \text { and } \max (a, b)=1\} .
$$

In other words, $U_{W}$ is the set of pairs of elements in $W$ such that one of them is 1 . An complete order $\geqq$ on $U_{W}$ is defined as

$$
\langle a, b\rangle \geqq\left\langle a^{\prime}, b^{\prime}\right\rangle \text { iff }\left\{\begin{array}{l}
\left(1 \geq a \geq a^{\prime} \& b=b^{\prime}=1\right) \vee \\
\left(a=1 \& a^{\prime}<1\right) \vee \\
\left(a=a^{\prime}=1 \& b^{\prime} \geq b\right)
\end{array}\right.
$$

Derivatives $\gg$ (strict preference) and $=$ (indifference) are also used. To distinguish the order on pairs $(\geqq)$ from the order on scalar $(\geq)$, we adopt the following convention: $\gg$ reads "higher", $\gg-$ "strictly higher" whereas $\geq$ reads "larger" and $>-$ "strictly larger". The special structure of $U_{W}$ i.e., $\langle a, b\rangle \in U_{W}$ implies $\max (a, b)=1$, allows a simplification of eq.(12) to

$$
\langle a, b\rangle \gg\left\langle a^{\prime}, b^{\prime}\right\rangle \quad \text { iff } \quad\left(a>a^{\prime}\right) \vee\left(b<b^{\prime}\right)
$$

We call $U_{W}$ equipped with the order $\gg$ as the binary utility scale based on $W$. This is a special case of a lexicographic order in Fishburn's sense. Although one index is used to resolve a tie in the other index, no index has precedence over the other. Intuitively, one can think of a binary utility level as a pair of indices reflecting two aspects of a quantity where the first number is the "positive" index and the second number is the "negative" index. Thus, eq. (13) says that a utility level $u$ is higher than utility level $u^{\prime}$ if either the positive index of $u$ is larger than the corresponding index of $u^{\prime}$ or the negative index of $u$ is smaller than that of $u^{\prime}$. Let us describe operations on pairs that are useful later on. Since the indices in $U_{W}$ are members of a linearly ordered scale $W$ where min and $\max$ are well defined, we can extend these operations for pairs. Suppose $\alpha, \beta, \gamma, \delta \in W$.

$$
\begin{array}{rll}
\min (\langle\alpha, \beta\rangle,\langle\gamma, \delta\rangle) & \stackrel{\text { def }}{=}\langle\min (\alpha, \gamma), \min (\beta, \delta)\rangle \\
\max (\langle\alpha, \beta\rangle,\langle\gamma, \delta\rangle) & \stackrel{\text { def }}{=}\langle\max (\alpha, \gamma), \max (\beta, \delta)\rangle
\end{array}
$$

Before we describe some properties of extended $\min$ and $\max$, it is important to note that these operations are just shorthands for doing pairwise minimization 


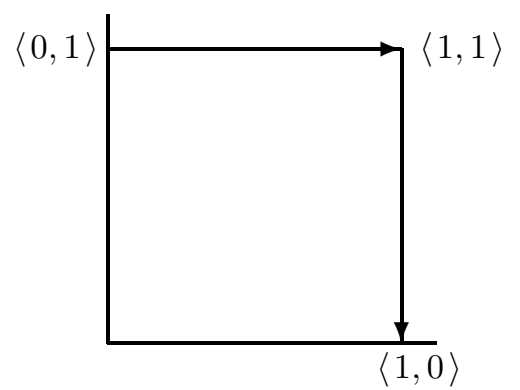

Figure 1: The binary utility scale $U_{W}$.

and maximization on scalars. They are not intended for finding infimum and supremum according to $\geqq$. In other words, for $u, v \in U_{W}$ we can deduce neither $\max (u, v) \geqq u$ nor $\max (u, v) \geqq v$. To do so we use sup notation. For $Z \subseteq U_{W}$

$$
\sup (Z) \in Z \text { and } \forall z \in Z \sup (Z) \geqq z
$$

We have $\sup (Z) \in Z$ because $W$ and therefore $U_{W}$ is assumed to be finite.

\section{Lemma 4}

(i) $U_{W}$ is closed under $\max$, i.e., if $u, u^{\prime} \in U_{W}$ then $\max \left(u, u^{\prime}\right) \in U_{W}$.

(ii) min, max are monotone on each argument, e.g., for $u, u^{\prime}, v, v^{\prime} \in U_{W}$ $\max (u, v) \geqq \max \left(u, v^{\prime}\right)$ if $v \geqq v^{\prime}$.

Proof: $(i)$ Let $u=\langle a, b\rangle, u^{\prime}=\left\langle a^{\prime}, b^{\prime}\right\rangle$. Since $u, u^{\prime} \in U_{W}$ implies $\max (a, b)=$ $\max \left(a^{\prime}, b^{\prime}\right)=1 . \max \left(u, u^{\prime}\right)=\left\langle\max \left(a, a^{\prime}\right), \max \left(b, b^{\prime}\right)\right\rangle$. We have

$$
\max \left\{\max \left(a, a^{\prime}\right), \max \left(b, b^{\prime}\right)\right\}=\max \left\{a, a^{\prime}, b, b^{\prime}\right\}=1 .
$$

(ii) Let $u=\langle a, b\rangle, v=\langle c, d\rangle, v^{\prime}=\left\langle c^{\prime}, d^{\prime}\right\rangle$. We have from $v \geqq v^{\prime}$ either $v=v^{\prime}$ i.e., $c=c^{\prime}$ and $d=d^{\prime}$, or $v \gg v^{\prime}$. In the first case, obviously $\max (u, v)=$ $\max \left(u, v^{\prime}\right)$. In the second case, by eq. (13), we have either $c>c^{\prime}$ or $d<d^{\prime}$. Moreover, $c>c^{\prime}$ implies $d^{\prime} \geq d$, and $d<d^{\prime}$ implies $c \geq c^{\prime}$ since $\max (c, d)=$ $\max \left(c^{\prime}, d^{\prime}\right)=1$. By definition of extended max in eq. (15) we have $\max (u, v)=$ $\langle\max (a, c), \max (b, d)\rangle$ and $\max \left(u, v^{\prime}\right)=\left\langle\max \left(a, c^{\prime}\right), \max \left(b, d^{\prime}\right)\right\rangle$. In both cases of $v \gg v^{\prime}$, we have $\max (a, c) \geq \max \left(a, c^{\prime}\right)$ and $\max \left(b, d^{\prime}\right) \geq \max (b, d)$. Thus the positive index of $\max (u, v)$ is larger than or equal to that of $\max \left(u, v^{\prime}\right)$ while the negative index of the former is smaller than or equal to one of the latter.

Let us call a function $u: X \rightarrow U_{W}$ that assigns utility for each prize in $X$ a basic utility assessment. We say that a basic utility assessment is consistent with $\succeq$ if for any pair $x, y \in X x \succeq y$ iff $u(x) \geqq u(y)$. For a given basic utility assessment $u$, let us define a utility function $P U: \Pi_{X} \rightarrow U_{W}$ as follows

$$
P U(\pi) \stackrel{\text { def }}{=} \max _{x \in X} \min (k(\pi(x)), u(x))
$$

where $k$ is a binary vector function $k=\left\langle k_{1}, k_{2}\right\rangle$ and each $k_{r}$ for $r=1,2$ is an order preserving mapping $V \rightarrow W$ such that $k_{r}(1)=1$ and $k_{r}(0)=0$. In 
addition, function $k$ is required to satisfy the following condition with respect to utility assignment $u$.

$$
k_{1}(V) \supseteq u^{L}(X) \text { and } k_{2}(V) \supseteq u^{R}(X)
$$

where $u^{L}(x)$ and $u^{R}(x)$ are the left and the right components of pair $u(x)$ respectively; $u^{L}(X)=\left\{u^{L}(x) \mid x \in X\right\}$ is the set of left values and $u^{R}(X)$ is the set of right values. This property is weaker than the requirement in the definitions of $Q U^{-}, Q U^{+}$that function $h$ defined in eq.(4) is an onto mapping since $u^{L}(X), u^{R}(X) \subseteq W$. The fact that $P U(\pi) \in U_{W}$ is easy to verify. Because $\exists x_{i} \in$ $X$ such that $\pi\left(x_{i}\right)=1, k\left(\pi\left(x_{i}\right)\right)=\langle 1,1\rangle$. So, $\min \left(k\left(\pi\left(x_{i}\right)\right), u\left(x_{i}\right)\right)=u\left(x_{i}\right) \in$ $U_{W}$. And therefore, $\max _{x \in X} \min (k(\pi(x)), u(x)) \in U_{W}$. Comparing $P U$ with the expected utility construct for probabilistic lottery $p, E U(p) \stackrel{\text { def }}{=} \sum_{x \in X} p(x) u(x)$, we see that operations $\max$ and $\min$ are used instead of addition and multiplication respectively. We call $P U(\pi)$ the expected qualitative utility of lottery $\pi$. In the next two lemmas, we describe some properties of $P U$.

Lemma $5 P U(x)=u(x)$.

Proof: Since we can write $x$ in a form of lottery $\pi_{x}$ such that $\pi_{x}(x)=1$ and $\pi_{x}\left(x^{\prime}\right)=0$ for $x^{\prime} \neq x$. Applying eq.(18) we have

$$
\begin{aligned}
P U\left(\pi_{x}\right) & =\max _{y \in X} \min \left(k\left(\pi_{x}(y)\right), u(y)\right) \\
& =\max \{\{\min (\langle 0,0\rangle, u(y)) \mid y \neq x\}, \min (\langle 1,1\rangle, u(x))\} \\
& =u(x) .
\end{aligned}
$$

From eq.(18), it is obvious that given $k$, a utility function is uniquely defined by a basic utility assessment. However, lemma 5 allows us to view eq. (18) not only as a constructive definition of $P U$ but also as property satisfied by a utility function $P U: \Pi_{X} \rightarrow U_{W}$ without referring to a basic utility assessment. Constructively, the former view is easy to understand but conceptually, the latter is more convenient. The two views will be used interchangeably.

Lemma 6 Assume $P U$ is defined as in eq. (18). Suppose $x$ is a prize such that $\bar{x} \succ x \succ \underline{x}$. Then there exists a canonical lottery $\sigma=[\alpha / \bar{x}, \beta / \underline{x}]$ such that $P U(\sigma)=P U(x)$.

Proof: Suppose for the basic utility assessment $u(\bar{x})=\left\langle\lambda_{1}, \mu_{1}\right\rangle, u(x)=\left\langle\lambda_{2}, \mu_{2}\right\rangle$ and $u(\underline{x})=\left\langle\lambda_{3}, \mu_{3}\right\rangle$. Since $u$ is assumed to be consistent and $\bar{x} \succ x \succ \underline{x}$, we have $\lambda_{1} \geq \lambda_{2} \geq \lambda_{3}$ and $\mu_{3} \geq \mu_{2} \geq \mu_{1}$. By condition eq. (19), we can choose $\alpha, \beta \in V$ such that $k_{1}(\alpha)=\lambda_{2}$ and $k_{2}(\beta)=\mu_{2}$. Since $\max \left(\lambda_{2}, \mu_{2}\right)=1$, we can choose $\alpha, \beta$ so that $\max (\alpha, \beta)=1$. Let us calculate $P U$ for $[\alpha / \bar{x}, \beta / \underline{x}]$

$$
\begin{aligned}
P U([\alpha / \bar{x}, \beta / \underline{x}]) & =\max \left\{\begin{array}{l}
\min \left(\left\langle k_{1}(\alpha), k_{2}(\alpha)\right\rangle,\left\langle\lambda_{1}, \mu_{1}\right\rangle\right), \\
\min \left(\left\langle k_{1}(\beta), k_{2}(\beta)\right\rangle,\left\langle\lambda_{3}, \mu_{3}\right\rangle\right)
\end{array}\right\} \\
& =\max \left\{\begin{array}{l}
\left\langle\min \left(k_{1}(\alpha), \lambda_{1}\right), \min \left(k_{2}(\alpha), \mu_{1}\right)\right\rangle, \\
\left\langle\min \left(k_{1}(\beta), \lambda_{3}\right), \min \left(k_{2}(\beta), \mu_{3}\right)\right\rangle
\end{array}\right\} \\
& =\left\langle k_{1}(\alpha), k_{2}(\beta)\right\rangle=\left\langle\lambda_{2}, \mu_{2}\right\rangle
\end{aligned}
$$


The chain of transformation is made possible by application of extended max, min and the facts that $\lambda_{1} \geq k_{1}(\alpha)=\lambda_{2} \geq \lambda_{3}$ and $\mu_{3} \geq k_{2}(\beta)=\mu_{2} \geq \mu_{1}$.

We now state and prove the main representation theorem.

Theorem $2 \succeq$ on $\Pi_{X}$ satisfies axioms $B 1$ through $B 4$ if and only if there exists a utility function $P U: \Pi_{X} \rightarrow U_{V}$ satisfying eq. (18) such that $\pi \succeq \pi^{\prime}$ if and only if $P U(\pi) \geqq P U\left(\pi^{\prime}\right) \forall \pi, \pi^{\prime} \in \Pi_{X}$.

Proof: Notice that the uncertainty scale $V$ is used for the linear order on which the utility scale is based.

$(\Rightarrow)$ Suppose $\succeq$ satisfies axioms $B 1$ through $B 4$. For $\pi_{1}, \pi_{2} \in \Pi_{X}$, let us assume $\pi_{1}\left(x_{i}\right)=\pi_{1 i}$ and $\pi_{2}\left(x_{i}\right)=\pi_{2 i}$ for $i \in\{1,2, \ldots n\}$. We will construct function $k$ and $P U$ such that $P U\left(\pi_{1}\right) \gg P U\left(\pi_{2}\right)$ if $\pi_{1} \succeq \pi_{2}$. Since the relation $\succeq$ on $\mathcal{C}$ is a pre-order, let us consider $\mathcal{C} / \sim$ - the set of equivalence classes. The equivalence class containing $\sigma$ is denoted by $\llbracket \sigma \rrbracket$. Without loss of generality, we assume a strict order $\bar{x}=x_{1}>x_{2}>\ldots>x_{n}=\underline{x}$. By $B 4, \llbracket x_{i} \rrbracket \neq \emptyset$ for all $i$. Let us choose an element from each equivalence class and call it the representative. For $\llbracket x_{1} \rrbracket,[1 / \bar{x}, 0 / \underline{x}]$ is selected, for $\llbracket x_{n} \rrbracket$ it is $[0 / \bar{x}, 1 / \underline{x}]$. For any other equivalence class, an element is selected arbitrarily. If $\left[\lambda_{i} / \bar{x}, \mu_{i} / \underline{x}\right]$ and $\left[\lambda_{j} / \bar{x}, \mu_{j} / \underline{x}\right]$ are not in one class, then either $\left[\lambda_{i} / \bar{x}, \mu_{i} / \underline{x}\right] \succ\left[\lambda_{j} / \bar{x}, \mu_{j} / \underline{x}\right]$ or $\left[\lambda_{j} / \bar{x}, \mu_{j} / \underline{x}\right] \succ\left[\lambda_{i} / \bar{x}, \mu_{i} / \underline{x}\right]$. If $\left[\lambda_{i} / \bar{x}, \mu_{i} / \underline{x}\right] \succ\left[\lambda_{j} / \bar{x}, \mu_{j} / \underline{x}\right]$, because of $B 2$, then either $\lambda_{i}>\lambda_{j}$ or $\mu_{i}<\mu_{j}$. We will proceed to define functions $k_{1}, k_{2}: V \rightarrow V$. For $\lambda, \mu \in V-\{0,1\}$

$$
\begin{aligned}
& k_{1}(\lambda) \stackrel{\text { def }}{=} \begin{cases}\lambda_{i} & \text { if }\left[\lambda_{i} / \bar{x}, 1 / \underline{x}\right] \text { is the representative of } \llbracket \lambda / \bar{x}, 1 / \underline{x} \rrbracket \\
1 & \text { if }[1 / \bar{x}, \mu / \underline{x}] \text { is the representative of } \llbracket \lambda / \bar{x}, 1 / \underline{x} \rrbracket\end{cases} \\
& k_{2}(\mu) \stackrel{\text { def }}{=} \begin{cases}\mu_{i} & \text { if }\left[1 / \bar{x}, \mu_{i} / \underline{x}\right] \text { is the representative of } \llbracket 1 / \bar{x}, \mu / \underline{x} \rrbracket \\
1 & \text { if }[\lambda / \bar{x}, 1 / \underline{x}] \text { is the representative of } \llbracket 1 / \bar{x}, \mu / \underline{x} \rrbracket\end{cases} \\
& k_{r}(0) \stackrel{\text { def }}{=} 0 \text { for } r=1,2 \\
& k_{r}(1) \stackrel{\text { def }}{=} 1 \text { for } r=1,2
\end{aligned}
$$

We will show $k_{1}(\alpha) \geq k_{1}(\beta)$ if $\alpha \geq \beta$. For $k_{2}$, the proof is similar. If $\beta=0$, by equation $(22), k_{1}(\beta)=0$. So, $k_{1}(\alpha) \geq k_{1}(\beta)$. If $\alpha=1$, by equation (23), $k_{1}(\alpha)=1$. Again, $k_{1}(\alpha) \geq k_{1}(\beta)$. Otherwise if $1>\alpha \geq \beta>0, k_{1}(\alpha), k_{1}(\beta)$ are determined by equation (20). By $B 2$, we have $[\alpha / \bar{x}, 1 / \underline{x}] \succeq[\beta / \bar{x}, 1 / \underline{x}]$. If those two canonical lotteries are in the same class $\llbracket \lambda_{i} / \bar{x}, \mu_{i} / \underline{x} \rrbracket$ then $k_{1}(\alpha)=$ $k_{1}(\beta)=\lambda_{i}$. If they are in different classes say $\llbracket \lambda_{i} / \bar{x}, \mu_{i} / \underline{x} \rrbracket$ and $\llbracket \lambda_{j} / \bar{x}, \mu_{j} / \underline{x} \rrbracket$, then $\left[\lambda_{i} / \bar{x}, \mu_{i} / \underline{x}\right] \succ\left[\lambda_{j} / \bar{x}, \mu_{j} / \underline{x}\right]$. By $B 2$ we have $\lambda_{i} \geq \lambda_{j}$. Thus $k_{1}(\alpha)=\lambda_{i} \geq$ $\lambda_{j}=k_{1}(\beta)$. Let us construct a basic utility assessment $u$ as follows $u(\bar{x})=\langle 1,0\rangle$, $u(\underline{x})=\langle 0,1\rangle$ and

$$
u\left(x_{i}\right)=\left\langle k_{1}\left(\lambda_{i}\right), k_{2}\left(\mu_{i}\right)\right\rangle \text { if } x_{i} \in \llbracket \lambda_{i} / \bar{x}, \mu_{i} / \underline{x} \rrbracket
$$

Thus, $u$ and $k$ satisfy condition listed in eq. (19). Assume $x_{i} \sim\left[\lambda_{i} / \bar{x}, \mu_{i} / \underline{x}\right]=\sigma_{i}$ for $i \in\{1,2, \ldots n\}$. By $B 3$, we have

$$
\pi_{r}=\left[\pi_{r 1} / x_{1}, \pi_{r 2} / x_{2}, \ldots \pi_{r n} / x_{n}\right] \sim\left[\pi_{r 1} / \sigma_{1}, \pi_{r 2} / \sigma_{2}, \ldots \pi_{r n} / \sigma_{n}\right] \text { for } r=1,2 .
$$


Let us give a name $\rho_{r}$ for $\left[\pi_{r 1} / \sigma_{1}, \pi_{r 2} / \sigma_{2}, \ldots \pi_{r n} / \sigma_{n}\right](r=1,2)$. We will show that $\rho_{r}$, which is a possibilistic mixture, is reduced to a canonical lottery. By definition of mixture

$$
\rho_{r}(\bar{x})=\max _{i \in\{1,2, \ldots n\}} \min \left(\pi_{r i}, \lambda_{i}\right) \text { and } \rho_{r}(\underline{x})=\max _{i \in\{1,2, \ldots n\}} \min \left(\pi_{r i}, \mu_{i}\right)
$$

and for all other $x \in X$

$$
\rho_{r}(x)=\max _{i \in\{1,2, \ldots n\}} \min \left(\pi_{r i}, 0\right)=0
$$

By $B 1$, from $\pi_{1} \succeq \pi_{2}$ we have $\rho_{1} \succeq \rho_{2}$, which means either $\rho_{1} \sim \rho_{2}$ or $\rho_{1} \succ \rho_{2}$. In case $\rho_{1} \sim \rho_{2}$, by definition of functions $k_{1}, k_{2}$ we have

$$
k_{1}\left(\rho_{1}(\bar{x})\right)=k_{1}\left(\rho_{2}(\bar{x})\right) \text { and } k_{2}\left(\rho_{1}(\underline{x})\right)=k_{2}\left(\rho_{2}(\underline{x})\right)
$$

In case $\rho_{1} \succ \rho_{2}$ by $B 2$ we have

$$
k_{1}\left(\rho_{1}(\bar{x})\right)>k_{1}\left(\rho_{2}(\bar{x})\right) \text { or } k_{2}\left(\rho_{1}(\underline{x})\right)<k_{2}\left(\rho_{2}(\underline{x})\right)
$$

Now let us calculate utilities of $\pi_{1}, \pi_{2}$

$$
\begin{aligned}
P U\left(\pi_{1}\right) & =\max _{i \in\{1,2, \ldots n\}}\left\{\min \left(\left\langle k_{1}\left(\pi_{1 i}\right), k_{2}\left(\pi_{1 i}\right)\right\rangle, u\left(x_{i}\right)\right)\right\} \\
& =\max _{i \in\{1,2, \ldots n\}}\left\{\min \left(\left\langle k_{1}\left(\pi_{1 i}\right), k_{2}\left(\pi_{1 i}\right)\right\rangle,\left\langle k_{1}\left(\lambda_{i}\right), k_{2}\left(\mu_{i}\right)\right\rangle\right)\right\} \\
& =\max _{i \in\{1,2, \ldots n\}}\left\{\left\langle\min \left(k_{1}\left(\pi_{1 i}\right), k_{1}\left(\lambda_{i}\right), \min \left(k_{2}\left(\pi_{1 i}\right), k_{2}\left(\mu_{i}\right)\right)\right\rangle\right\}\right. \\
& =\max _{i \in\{1,2, \ldots n\}}\left\{\left\langle\left(k_{1}\left(\min \left(\pi_{1 i}, \lambda_{i}\right)\right), k_{2}\left(\min \left(\pi_{1 i}, \mu_{i}\right)\right)\right\rangle\right\}\right. \\
& =\left\langle k_{1}\left(\max _{i \in\{1,2, \ldots n\}}\left\{\min \left(\pi_{1 i}, \lambda_{i}\right)\right\}\right), k_{2}\left(\max _{i \in\{1,2, \ldots n\}}\left\{\min \left(\pi_{1 i}, \mu_{i}\right)\right\}\right)\right\rangle \\
& =\left\langle k_{1}\left(\rho_{1}(\bar{x})\right), k_{2}\left(\rho_{1}(\underline{x})\right)\right\rangle
\end{aligned}
$$

The transformation chain is made possible by the assumption about $u\left(x_{i}\right)$, the properties of min, $\max$, the monotonicity of $k_{1}, k_{2}$ and equation (26). Recall that if $\pi_{1} \succeq \pi_{2}$ then either condition (28) or (29) must hold. In either case, that implies $\left\langle k_{1}\left(\rho_{1}(\bar{x})\right), k_{2}\left(\rho_{1}(\underline{x})\right)\right\rangle \geqq\left\langle k_{1}\left(\rho_{2}(\bar{x})\right), k_{2}\left(\rho_{2}(\underline{x})\right)\right\rangle$. Thus, $P U\left(\pi_{1}\right) \geqq P U\left(\pi_{2}\right)$.

$(\Leftarrow)$ For a given consistent basic utility assessment $u: X \rightarrow \bar{U}_{V}$, order preserving functions $k_{1}, k_{2}$ such that $k_{r}(0)=0, k_{r}(1)=1$, a function $P U$ : $\Pi_{X} \rightarrow U_{V}$ is defined as in (18). We will show that order $\succeq_{P U}$ on $\Pi_{X}$ induced by $P U$ i.e., $\pi \succeq_{P U} \pi^{\prime}$ iff $P U(\pi) \geqq P U\left(\pi^{\prime}\right)$ satisfies axioms $B 1$ through $B 4$.

$B 1$ is satisfied because the order on $U_{V}$ is reflexive, transitive and complete. We turn to $B 2$. Suppose $\sigma=[\lambda / \bar{x}, \mu / \underline{x}], \sigma^{\prime}=\left[\lambda^{\prime} / \bar{x}, \mu^{\prime} / \underline{x}\right]$ are two canonical lotteries that satisfy one of three conditions (1) $\left(\lambda \geq \lambda^{\prime} \& \mu=\mu^{\prime}=1\right)$, (2) $\left(\lambda=\lambda^{\prime}=1 \& \mu^{\prime} \geq \mu\right)$ or $(3)\left(\lambda=1>\lambda^{\prime}\right)$. We have to show $P U(\sigma) \geqq P U\left(\sigma^{\prime}\right)$. Let us assume $u(\bar{x})=\langle\bar{\lambda}, \bar{\mu}\rangle$ and $u(\underline{x})=\langle\underline{\lambda}, \underline{\mu}\rangle$. Since $u$ is consistent and $\bar{x} \succ \underline{x}$ we have either $(\bar{\lambda}>\underline{\lambda} \& \bar{\mu}=\underline{\mu})$ or $(\bar{\lambda}=\underline{\lambda} \& \bar{\mu}<\underline{\mu})$ or $(1=\bar{\lambda}>\underline{\lambda} \& \bar{\mu}<\underline{\mu}=1)$. 
Applying definition (18), we have

$$
\begin{aligned}
& P U(\sigma) \\
& \quad=\max \left\{\min \left(\left\langle k_{1}(\lambda), k_{2}(\lambda)\right\rangle,\langle\bar{\lambda}, \bar{\mu}\rangle\right), \min \left(\left\langle k_{1}(\mu), k_{2}(\mu)\right\rangle,\langle\underline{\lambda}, \underline{\mu}\rangle\right)\right\} \\
& \quad=\max \left\{\left\langle\min \left(k_{1}(\lambda), \bar{\lambda}\right), \min \left(k_{2}(\lambda), \bar{\mu}\right)\right\rangle,\left\langle\min \left(k_{1}(\mu), \underline{\lambda}\right), \min \left(k_{2}(\mu), \underline{\mu}\right)\right\rangle\right\} \\
& \quad=\left\langle\max \left\{\min \left(k_{1}(\lambda), \bar{\lambda}\right), \min \left(k_{1}(\mu), \underline{\lambda}\right)\right\}, \max \left\{\min \left(k_{2}(\lambda), \bar{\mu}\right), \min \left(k_{2}(\mu), \underline{\mu}\right)\right\}\right\rangle
\end{aligned}
$$

Thus, the positive (left) $\rho_{l}$ and the negative indices (right) $\rho_{r}$ of $P U(\sigma)$ and $P U\left(\sigma^{\prime}\right)$ are

$$
\begin{aligned}
\rho_{l}(\sigma) & =\max \left\{\min \left(k_{1}(\lambda), \bar{\lambda}\right), \min \left(k_{1}(\mu), \underline{\lambda}\right)\right\} \\
\rho_{l}\left(\sigma^{\prime}\right) & =\max \left\{\min \left(k_{1}\left(\lambda^{\prime}\right), \bar{\lambda}\right), \min \left(k_{1}\left(\mu^{\prime}\right), \underline{\lambda}\right)\right\} \\
\rho_{r}(\sigma) & =\max \left\{\min \left(k_{2}(\lambda), \bar{\mu}\right), \min \left(k_{2}(\mu), \underline{\mu}\right)\right\} \\
\rho_{r}\left(\sigma^{\prime}\right) & =\max \left\{\min \left(k_{2}\left(\lambda^{\prime}\right), \bar{\mu}\right), \min \left(k_{2}\left(\mu^{\prime}\right), \underline{\mu}\right)\right\}
\end{aligned}
$$

In case (1) when $\lambda \geq \lambda^{\prime}$ and $\mu=\mu^{\prime}=1$, since operations used are all order preserving, we conclude $\rho_{l}(\sigma) \geq \rho_{l}\left(\sigma^{\prime}\right)$. In case (2) when $\lambda=\lambda^{\prime}=1$ and $\mu^{\prime} \geq \mu$, we have $\rho_{r}\left(\sigma^{\prime}\right) \leq \rho_{r}(\sigma)$. In the last case, from $1=\lambda>\lambda^{\prime}$ we also have $\mu^{\prime} \leq \mu$. By the same argument we will have both $\rho_{l}(\sigma) \geq \rho_{l}\left(\sigma^{\prime}\right)$ and $\rho_{r}\left(\sigma^{\prime}\right) \geq \rho_{r}(\sigma)$. By definition given in eq. (12) we have $P U(\sigma) \geqq P U\left(\sigma^{\prime}\right)$ and that means $\sigma \succeq_{P U} \sigma^{\prime}$. Thus $B 2$ is satisfied. Suppose $\pi_{1} \sim_{P U} \pi_{2}$. We have $P U\left(\pi_{1}\right)=P U\left(\pi_{2}\right)$. By definition (18), we will have $P U\left(\left[\lambda / \pi_{1}, \mu / \pi\right]\right)=P U\left(\left[\lambda / \pi_{2}, \mu / \pi\right]\right)$. This means $\left[\lambda / \pi_{1}, \mu / \pi\right] \sim_{P U}\left[\lambda / \pi_{2}, \mu / \pi\right]$. Thus, $\succeq_{P U}$ satisfies $B 3$. Finally, the existence of basic utility assessment $u$ and lemma 6 guarantee satisfaction of $B 4$.

This representation theorem summarizes the utility theory for possibility theory. Among other things this theorem shows that axiom system $\mathcal{S}$ is free from inconsistency. The main corollary is the decision rule, which we call maximization of expected qualitative utility (MEQU): facing a choice among lotteries in the set $\left\{L_{1}, L_{2}, \ldots L_{n}\right\}$, a rational decision maker should choose

$$
L^{*}=\arg \sup _{i \in\{1,2, \ldots n\}} P U\left(L_{i}\right)
$$

We illustrate MEQU with a numerical example.

Example 2. In this example, sets $X, V$ and $\pi_{1}, \pi_{2}, \pi_{3}$ are the same as used in example 1. $U_{V}=\{\langle 0,1\rangle,\langle .5,1\rangle,\langle .7,1\rangle,\langle 1,1\rangle,\langle 1, .7\rangle,\langle 1, .5\rangle,\langle 1,0\rangle\}$. We will consider three cases of revealed indifference relation between the prizes and canonical lotteries.

Case 1. In this case, $\bar{x} \sim[1 / \bar{x}, 0 / \underline{x}], x_{2} \sim[1 / \bar{x}, .5 / \underline{x}], x_{3} \sim[1 / \bar{x}, .7 / \underline{x}]$ and $\underline{x} \sim[1 / \bar{x}, 1 / \underline{x}]$. This corresponds to a pessimistic decision maker. Utility assignment for prizes is as follows $u(\bar{x})=\langle 1,0\rangle, u\left(x_{2}\right)=\langle 1, .5\rangle, u\left(x_{3}\right)=\langle 1, .7\rangle$ and $u(\underline{x})=\langle 1,1\rangle$. We choose functions $k_{1}(0)=0$ and $k_{1}(v)=1$ for $v \in V-0$; $k_{2}(v)=v$ for $v \in V$. We calculate expected qualitative utility for $\pi_{i}, i=1,2,3$.

$$
P U\left(\pi_{1}\right)=\max \left\{\begin{array}{l}
\min (k(.7), u(\bar{x})) \\
\min \left(k(1), u\left(x_{2}\right)\right) \\
\min \left(k(.5), u\left(x_{3}\right)\right) \\
\min (k(.5), u(\underline{x}))
\end{array}\right\}=\max \left\{\begin{array}{l}
\min (\langle 1, .7\rangle,\langle 1,0\rangle) \\
\min (\langle 1,1\rangle,\langle 1, .5\rangle) \\
\min (\langle 1, .5\rangle,\langle 1, .7\rangle) \\
\min (\langle 1, .5\rangle,\langle 1,1\rangle)
\end{array}\right\}
$$




$$
=\max \left\{\begin{array}{l}
\langle 1,0\rangle \\
\langle 1, .5\rangle \\
\langle 1, .5\rangle \\
\langle 1, .5\rangle
\end{array}\right\}=\langle 1, .5\rangle
$$

Similarly we calculate $P U\left(\pi_{2}\right)=\langle 1,1\rangle$ and $P U\left(\pi_{3}\right)=\langle 1,1\rangle$. Thus $\pi_{1} \succ \pi_{2} \sim \pi_{3}$. This is the same preference order as in case 1 of example 1 .

Case 2. In this case revealed indifference is $\bar{x} \sim[1 / \bar{x}, 1 / \underline{x}], x_{2} \sim[.7 / \bar{x}, 1 / \underline{x}]$, $x_{3} \sim[.5 / \bar{x}, 1 / \underline{x}]$ and $\underline{x} \sim[0 / \bar{x}, 1 / \underline{x}]$. Utility assignment for prizes is as follows $u(\bar{x})=\langle 1,1\rangle, u\left(x_{2}\right)=\langle .7,1\rangle, u\left(x_{3}\right)=\langle .5,1\rangle$ and $u(\underline{x})=\langle 0,1\rangle$. We choose functions $k_{1}(v)=v$ for $v \in V$ and $k_{2}(0)=0$ and $k_{2}(v)=1$ for $v \in V-0$. We calculate expected qualitative utility for $\pi_{i}, i=1,2,3$.

$$
\begin{aligned}
P U\left(\pi_{1}\right) & =\max \left\{\begin{array}{l}
\min (k(.7), u(\bar{x})) \\
\min \left(k(1), u\left(x_{2}\right)\right) \\
\min \left(k(.5), u\left(x_{3}\right)\right) \\
\min (k(.5), u(\underline{x}))
\end{array}\right\}=\max \left\{\begin{array}{l}
\min (\langle .7,1\rangle,\langle 1,1\rangle) \\
\min (\langle 1,1\rangle,\langle .7,1\rangle) \\
\min (\langle .5,1\rangle,\langle .5,1\rangle) \\
\min (\langle .5,1\rangle,\langle 0,1\rangle)
\end{array}\right\} \\
= & \max \left\{\begin{array}{l}
\langle .7,1\rangle \\
\langle .7,1\rangle \\
\langle .5,1\rangle \\
\langle 0,1\rangle
\end{array}\right\}=\langle .7,1\rangle
\end{aligned}
$$

We also calculate $P U\left(\pi_{2}\right)=\langle .7,1\rangle$ and $P U\left(\pi_{3}\right)=\langle 1,1\rangle$. Thus $\pi_{3} \succ \pi_{1} \sim \pi_{2}$. This is the same preference order as in case 2 of example 1 .

Case 3. In this case revealed indifference is $\bar{x} \sim[1 / \bar{x}, 0 / \underline{x}], x_{2} \sim[1 / \bar{x}, .5 / \underline{x}]$, $x_{3} \sim[.7 / \bar{x}, 1 / \underline{x}]$ and $\underline{x} \sim[0 / \bar{x}, 1 / \underline{x}]$. Utility assignment for prizes is as follows $u(\bar{x})=\langle 1,0\rangle, u\left(x_{2}\right)=\langle 1, .5\rangle, u\left(x_{3}\right)=\langle .7,1\rangle$ and $u(\underline{x})=\langle 0,1\rangle$. Functions $k_{1}, k_{2}$ are identity i.e., $k(v)=\langle v, v\rangle$ for $v \in V$. We calculate expected utility for $\pi_{1}, \pi_{2}$ and $\pi_{3}$

$$
\begin{aligned}
P U\left(\pi_{1}\right) & =\max \left\{\begin{array}{l}
\min (k(.7), u(\bar{x})) \\
\min \left(k(1), u\left(x_{2}\right)\right) \\
\min \left(k(.5), u\left(x_{3}\right)\right) \\
\min (k(.5), u(\underline{x}))
\end{array}\right\}=\max \left\{\begin{array}{l}
\min (\langle .7, .7\rangle,\langle 1,0\rangle) \\
\min (\langle 1,1\rangle,\langle 1, .5\rangle) \\
\min (\langle .5, .5\rangle,\langle .7,1\rangle) \\
\min (\langle .5, .5\rangle,\langle 0,1\rangle)
\end{array}\right\} \\
& =\max \left\{\begin{array}{l}
\langle .7,0\rangle \\
\langle 1, .5\rangle \\
\langle .5, .5\rangle \\
\langle 0, .5\rangle
\end{array}\right\}=\langle 1, .5\rangle
\end{aligned}
$$

Similarly we have $P U\left(\pi_{2}\right)=\langle .7,1\rangle$ and $P U\left(\pi_{3}\right)=\langle 1,1\rangle$. So, $P U$ ranks $\pi_{1} \succ$ $\pi_{3} \succ \pi_{2}$.

\section{A Comparison of Pessimistic, Optimistic and Expected Utilities}

In this section, we will compare the 3 axiomatic systems described in previous sections. As already noted, the main difference is in the second axioms, so the 
discussion is focused on them.

First, we have some comments on uncertainty aversion (pessimism) and uncertainty attraction (optimism) axioms. Since we have representation theorems for each system, discussion can be carried out either in terms of axioms or in terms of utility functions. We argue that those axioms should be removed in favor of a more intuitive axiom $B 2$ that imposes an order on set of canonical lotteries. Finally, we prove a theorem showing that the pessimistic and optimistic systems of axioms are special cases of the generalized system proposed in section 3 .

The adjectives "pessimistic" and "optimistic", used for $\mathcal{S}_{P}, \mathcal{S}_{O}$, apparently refer to the opposite requirement of axioms $A 2^{-}$and $A 2^{+}$. If $\pi_{1} \geq \pi_{2}$ in numerical sense, i.e., $\pi_{1}\left(x_{i}\right) \geq \pi_{2}\left(x_{i}\right) \forall i$, then $A 2^{-}$requires $\pi_{2} \succeq \pi_{1}$ while $A 2^{+}$ requires $\pi_{1} \succeq \pi_{2}$. $A 2^{-}$and $A 2^{+}$are uncertainty attitude axioms. The former is "uncertainty aversion" and the latter is "uncertainty attraction".

First, we comment about the terminology. Since $\mathcal{S}_{P}, \mathcal{S}_{O}$ are presented in the style of von Neumann and Morgenstern [18], it is appropriate to recall similar terms "risk aversion" and "risk attraction". In the classical linear utility theory, risk aversion (attraction) refers to the concavity (convexity) of the basic utility assessment. In other words, risk aversion (risk attraction) is a property ascribed to individual utility functions. They are not a property of the utility theory. Although possibility theory deals with uncertainty, not risk, we feel that this difference does not warrant the use of different decision theories in different psychological states as implied by $\mathcal{S}_{P}$ and $\mathcal{S}_{O}$. The term risk is used, according to well accepted dichotomy, as uncertainty representable by a probability distribution. For example, it raises the obvious questions of how a decision maker would classify herself as either "optimistic" or "pessimistic" and what would happen if she could not be classified categorically as neither. The indifference relation revealed in case 3 of example 2 illustrates the latter situation.

In possibilistic literature ([6]), it is established that inequality of the form $\pi_{1} \geq \pi_{2}$ is an informational relationship. It says that $\pi_{2}$ is more specific than $\pi_{1}$. In other words, it says that $\pi_{2}$ contains more information than $\pi_{1}$ does. From that perspective, we fail to see the rationale for equating informational relationship with preferential relationship $\succeq$. Although information has its own value, informational value per se rarely serves as a decision criterion. For example, decision making under uncertainty is mostly guided by von Neumann and Morgenstern's linear utility theory rather than by Shannon's information theory.

Let us analyze the pessimistic system in greater details with a understanding that a similar analysis can be done for the optimistic system. Let us consider the following example. A decision maker faces a choice between two lotteries $\pi_{1}=[1 / \bar{x}, 1 / \underline{x}]$ and $\pi_{2}=\underline{x}$. In other words, $\pi_{1}$ is a possibilistic distribution on $X$ such that $\pi_{1}(\bar{x})=\pi_{1}(\underline{x})=1, \pi_{1}(x)=0$ for all other $x$ and $\pi_{2}(\underline{x})=1, \pi_{2}(x)=0$ for all other $x$. According to possibility theory [6], $\pi_{1}$ describes a situation where we have knowledge to exclude all prizes except $\bar{x}$ and $\underline{x}$. Moreover, the occurrence of either of the two prizes is equally possible. $\pi_{2}$ describes a situation of complete knowledge where all but $\underline{x}$ are excluded. Because something is going 
to happen, $\pi_{2}$ is equivalent to saying that $\underline{x}$ obtains for sure. Axiom $A 2^{-}$will force the decision maker to consider $\pi_{2}$ is at least as good as $\pi_{1}$. To be exact, a complete calculation would show that $\pi_{1} \sim \pi_{2}$. In other words, if we were to adopt $\mathcal{S}_{P}$, we would have to be indifferent between a worst prize for sure and a uncertain outcome in which we have reason to believe that the best prize is as likely as the worst one. We consider such a preference intuitively unreasonable.

Next we will show that the pessimism axiom forces the continuity axiom $A 4^{-}$to have an unnecessarily restricted form. To simplify the discussion, we need some notations. The set of canonical lotteries $\mathcal{C}=\{[\lambda / \bar{x}, \mu / \underline{x}] \mid \lambda, \mu \in$ $V, \max (\lambda, \mu)=1\}$ can be divided into two segments

$$
\mathcal{C}^{-} \stackrel{\text { def }}{=}\{[1 / \bar{x}, \mu / \underline{x}] \mid \mu \in V\} \text { and } \mathcal{C}^{+} \stackrel{\text { def }}{=}\{[\lambda / \bar{x}, 1 / \underline{x}] \mid \lambda \in V\} .
$$

We will call $\mathcal{C}^{-}$the gain half of the set of canonical lotteries for the possibility of getting the best prize is higher than or equal to the possibility of getting the worst prize and $\mathcal{C}^{+}$- the loss half. These terms are used relatively to the fair lottery $[1 / \bar{x}, 1 / \underline{x}]$. "Gain" does not mean a positive monetary value nor "loss" means a negative monetary value. We have $\mathcal{C}=\mathcal{C}^{-} \cup \mathcal{C}^{+}$and $\mathcal{C}^{-} \cap \mathcal{C}^{+}=$ $[1 / \bar{x}, 1 / \underline{x}]$. Using this notation, axioms $A 4^{-}$and $A 4^{+}$can be restated as follows $\forall \pi \in \Pi_{X}, \exists \sigma \in \mathcal{C}^{-}$such that $\pi \sim \sigma$, and $\forall \pi \in \Pi_{X}, \exists \sigma \in \mathcal{C}^{+}$such that $\pi \sim \sigma$, respectively. We will show that these axioms can be weakened, without any effect on the results, by requiring $\forall x \in X$ in place of $\forall \pi \in \Pi_{X}$ i.e.,

$$
\begin{gathered}
\left(B 4^{-}\right) \forall x \in X, \exists \sigma \in \mathcal{C}^{-} \quad x \sim \sigma . \\
\left(B 4^{+}\right) \forall x \in X, \exists \sigma \in \mathcal{C}^{+} \quad x \sim \sigma .
\end{gathered}
$$

\section{Lemma 7}

(i) A relation $\succeq^{-}$satisfies $A 1^{-}, A 2^{-}, A 3^{-}$and $A 4^{-}$iff it satisfies $A 1^{-}, A 2^{-}$, $A 3^{-}$and $B 4^{-}$.

(ii) A relation $\succeq^{+}$satisfies $A 1^{+}, A 2^{+}, A 3^{+}$and $A 4^{+}$iff it satisfies $A 1^{+}, A 2^{+}$, $\mathrm{A3}^{+}$and $B 4^{+}$.

Proof: We will prove $(i)$. The proof of $(i i)$ is similar.

$(\Rightarrow)$ is obvious because $X \subset \Pi_{X}$.

$(\Leftarrow)$ By $B 4^{-}$, each $x_{i} \in X$ is indifferent to some canonical lottery $\sigma_{i}=\left[1 / \bar{x}, \mu_{i} / \underline{x}\right]$ For any $\pi \in \Pi_{X}$, we can write $\pi=\left[\pi_{1} / x_{1}, \ldots \pi_{n} / x_{n}\right]$. By $A 3^{-}$, we have $\pi \sim$ $\left[\pi_{1} / \sigma_{1}, \ldots \pi_{n} / \sigma_{n}\right]$. By definition of possibilistic mixture we have $\left[\pi_{1} / \sigma_{1}, \pi_{2} / \sigma_{2}, \ldots\right.$ $\left.\pi_{n} / \sigma_{n}\right] \sim\left[\rho_{1} / \bar{x}, \rho_{2} / \underline{x}\right]$ where

$$
\rho_{1}=\max _{i \in\{1,2, \ldots n\}}\left\{\min \left(\pi_{i}, 1\right)\right\} \quad \rho_{2}=\max _{i \in\{1,2, \ldots n\}}\left\{\min \left(\pi_{i}, \mu_{i}\right)\right\}
$$

Since $\max _{i \in\{1,2, \ldots n\}} \pi_{i}=1$, we have $\rho_{1}=1$ Thus, $A 4^{-}$is satisfied.

A natural question arises: would a simple replacement of $A 4^{-}$by $B 4$ solve the problem? The answer is no, if one insists on keeping $A 2^{-}$. Had $A 4^{-}$been substituted by $B 4 \forall x \in X, \exists \sigma \in \mathcal{C} x \sim \sigma$, we would still have $\underline{x} \sim[1 / \bar{x}, 1 / \underline{x}]$, 
because $[1 / \bar{x}, 1 / \underline{x}]$ was the minimal element in $\mathcal{C}$ according to $A 2^{-}$. Moreover, the substitution would ruin the representation theorem 1 . To show this problem, we need the following lemma

Lemma 8 Suppose $\pi_{1} \succeq \pi_{2}$ then

$$
\begin{aligned}
& Q U^{-}\left(\left[\lambda / \pi_{1}, 1 / \pi_{2}\right]\right)=Q U^{-}\left(\pi_{2}\right) \\
& Q U^{+}\left(\left[1 / \pi_{1}, \mu / \pi_{2}\right]\right)=Q U^{+}\left(\pi_{1}\right) .
\end{aligned}
$$

Proof: We will prove (33). The proof of (34) is similar. We have by eq. (5)

$$
Q U^{-}\left(\left[\lambda / \pi_{1}, 1 / \pi_{2}\right]\right)=\min \left\{\max \left(n h(\lambda), Q U^{-}\left(\pi_{1}\right)\right), \max \left(n h(1), Q U^{-}\left(\pi_{2}\right)\right)\right\}
$$

By definition of function $n h$ eqs. $(3,4), n h(1)=0$. So, $\max \left(n h(1), Q U^{-}\left(\pi_{2}\right)\right)=$ $Q U^{-}\left(\pi_{2}\right)$. Since $\pi_{1} \succeq \pi_{2}, Q U^{-}\left(\pi_{1}\right) \geq Q U^{-}\left(\pi_{2}\right)$. So, we have $\max \left(n h(\lambda), Q U^{-}\left(\pi_{1}\right)\right)$ $\geq Q U^{-}\left(\pi_{2}\right)$. By equation (35), we have $Q U^{-}\left(\left[\lambda / \pi_{1}, 1 / \pi_{2}\right]\right)=Q U^{-}\left(\pi_{2}\right)$.

Now, let us assume for a moment $A 4^{-}$is replaced by $B 4$ in $\mathcal{S}_{P}$. So, for some $x^{\prime} \succ \underline{x}$ we have $x^{\prime} \sim[\lambda / \bar{x}, 1 / \underline{x}]$. By lemma 8, $Q U^{-}([\lambda / \bar{x}, 1 / \underline{x}])=Q U^{-}(\underline{x})$. Since $x^{\prime} \succ \underline{x}$, we conclude that $Q U^{-}$does not represent $\succeq$.

Furthermore, we argue that $A 4^{-}$contradicts intuitively the pessimistic spirit of $A 2^{-}$. $A 4^{-}$requires that any lottery is equivalent to some canonical lottery where the best prize $(\bar{x})$ is more possible or as possible as the least desirable prize $(\underline{x})$. This fact is also implied from the weakened version $B 4^{-}$. In that sense, if a decision maker holds a preference satisfying the pessimistic system, she must be, in fact, too optimistic because in her uncertain universe the best prize always has higher possibility of realization than the worst prize.

We have just analyzed the problems with the pessimistic $\mathcal{S}_{P}$. A similar critique can be made against the optimistic $\mathcal{S}_{O}$. This analysis leads to the conclusion that uncertainty attitude axioms $A 2^{-}, A 2^{+}$are the reasons behind the problems of systems $\mathcal{S}_{P}$ and $\mathcal{S}_{O}$. We propose a remedy in axiom system $\mathcal{S}$. Instead of $A 2^{-}, A 2^{+}$we have $B 2$ that imposes an order on canonical lotteries.

It is important to note that $B 2$ can be justified without invoking information consideration. Since canonical lottery $\sigma \in \mathcal{C}$ is a possibility distribution on $X$ such that $\sigma(\bar{x})=\lambda, \sigma(\underline{x})=\mu$ and $\sigma(x)=0$ for all other $x \in X$, the possibility degree and the necessity degree assigned by $\sigma$ to $\bar{x}$ are $\lambda$ and $1-\mu$. Because different canonical lotteries have exactly the same prizes, the intuitive preference boils down to comparing how sure the prizes will be realized. Obviously, the higher the confidence of getting the most desirable prize the better, provided that the confidence of getting the worst prize is the same or weaker. In possibility theory, the highest confidence - completely sure - is expressed by necessity degree equal to 1 . $N(\bar{x})=1$ means $\lambda=1$ and $\mu=0$. The confidence level is decreasing when necessity falling to 0 . This movement corresponds to $\mu$ increasing to 1 . Before necessity degree becomes 0 , the possibility is always 1 . Once necessity equals 0 , the confidence level can drop further with the falling of possibility degree from 1 to 0 . The least confidence level is when the possibility degree is 0 i.e. $\lambda=0$ and $\mu=1$. 
In [11] we propose a theoretically more interesting justification for $B 2$ based on the concept of first degree stochastic dominance.

Axiom $B 2$ (order between canonical lotteries) is able to unify and extend the effects achieved by two contradictory premises namely pessimism and optimism. It is straightforward to verify the following lemma

Lemma 9 Let $\succeq^{-}, \succeq^{+}$and $\succeq$ denote, respectively, the order relations on $\mathcal{C}^{-}$, $\mathcal{C}^{+}$and $\mathcal{C}$ imposed by $A 2^{-}, A 2^{+}$and $B 2$. We have $\succeq=\succeq^{-} \cup \succeq^{+} \cup\left(\mathcal{C}^{-} \times \mathcal{C}^{+}\right)$

The lemma shows that $\succeq^{-}\left(\succeq^{+}\right)$is $\succeq$ restricted to $\mathcal{C}^{-}\left(\mathcal{C}^{+}\right)$. This relationship has a direct consequence to the discrimination power of axiom systems (utility functions) because, as noted, each lottery is equivalent to some canonical lottery. Roughly, if the uncertainty scale $V$ has cardinality $n, \mathcal{S}_{P}\left(\mathcal{S}_{O}\right)$ divides $\Pi_{X}$ into $n$ equivalence classes while $\mathcal{S}$ makes $2 n-1$ classes.

We have the following theorem that states precisely the relationship between systems $\mathcal{S}_{P}, \mathcal{S}_{O}$ and $\mathcal{S}$.

\section{Theorem 3}

(i) $\mathcal{S}_{P} \models \mathcal{S}$

(ii) $\mathcal{S}_{O}=\mathcal{S}$

(iii) $\mathcal{S}, B 4^{-} \models \mathcal{S}_{P}$

(iv) $\mathcal{S}, B 4^{+} \models \mathcal{S}_{O}$

Proof: We will prove $(i)$ and $(i i i)$. The proofs of $(i i)$ and $(i v)$ are similar.

(i) Assume $A 1^{-}$through $A 4^{-}$are satisfied, since $B 1$ is the same as $A 1^{-}$and $B 3$ is the same as $A 3^{-}$, we are left to prove that $B 2$ and $B 4$ are also satisfied. From $A 4^{-}$for each $\pi \in \Pi_{X}, \exists \sigma \in \mathcal{C}^{-} \pi \sim \sigma$. Obviously, $X \subseteq \Pi_{X}$ and $\mathcal{C}^{-} \subseteq \mathcal{C}$, so $B 4$ is also satisfied. And finally, we will show that $\mathcal{S}_{P} \models B 2$. Assume that $\succeq$ satisfies $\mathcal{S}_{P}$. For two canonical lotteries $\sigma=[\lambda / \bar{x}, \mu / \underline{x}]$ and $\sigma^{\prime}=\left[\lambda^{\prime} / \bar{x}, \mu^{\prime} / \underline{x}\right]$. We want to show

$$
\sigma \succeq \sigma^{\prime} \text { if }\left\{\begin{array}{l}
1 \geq \lambda \geq \lambda^{\prime} \& \mu=\mu^{\prime}=1 \quad(a) \\
\lambda=1 \& \lambda^{\prime}<1 \\
\lambda=\lambda^{\prime}=1 \& \mu^{\prime} \geq \mu
\end{array}\right.
$$

Observe that by theorem 1 and lemma 8 , to a pessimistic decision maker, when the possibility associated with a less desirable prize is 1 then the lottery is indifferent to that prize. If $\mu=1$ then $\sigma \sim \underline{x} \sim[1 / \bar{x}, 1 / \underline{x}]$. If $\mu^{\prime}=1$ then $\sigma^{\prime} \sim \underline{x} \sim[1 / \bar{x}, 1 / \underline{x}]$. In case $(a)$ when $\mu=\mu^{\prime}=1$, we have $\sigma \sim \underline{x} \sim \sigma^{\prime}$. Thus, $\sigma \succeq \sigma^{\prime}$. In case $(b)$, since $\max \left(\lambda^{\prime}, \mu^{\prime}\right)=1$ from $\lambda^{\prime}<1$ we have $\mu^{\prime}=1$. Therefore, $\sigma^{\prime} \sim[1 / \bar{x}, 1 / \underline{x}]$. By axiom $A 2^{-}$we have $\sigma \succeq[1 / \bar{x}, 1 / \underline{x}]$. From transitivity, $\sigma \succeq \sigma^{\prime}$. In case $(c)$, since $\lambda=\lambda^{\prime}=1$ and $\mu^{\prime} \geq \mu$, by $A 2^{-}$we have $\sigma \succeq \sigma^{\prime}$.

(iii) Note that since $B 4^{-} \models B 4$, the set of axioms $\mathcal{S} \cup B 4^{-}$is effectively one that is obtained by substituting $B 4$ by $B 4^{-}$. We have to show that if $B 1, B 2, B 3$ and $B 4^{-}$are satisfied so are $A 1^{-}, A 2^{-}, A 3^{-}$and $A 4^{-}$. Again, we do not have to worry about $A 1^{-}$and $A 3^{-}$since they are identical to $B 1$ and $B 3$. We will show that $A 4^{-}$is satisfied. By $B 4^{-}$we assume $x_{i} \sim \sigma_{i}=\left[1 / \bar{x}, \mu_{i} / \underline{x}\right]$ for $i \in\{1,2, \ldots n\}$. We will show that $\forall \pi \in \Pi_{X}, \exists \sigma \in \mathcal{C}^{-} \pi \sim \sigma$. By $B 3$, 
$\pi \sim\left[\pi\left(x_{1}\right) / \sigma_{1}, \pi\left(x_{2}\right) / \sigma_{2} \ldots \pi\left(x_{n}\right) / \sigma_{n}\right]$. Applying the definition of mixture (1) for the right hand side, say $\rho$, of the indifference

$$
\rho(\bar{x})=\max _{i \in\{1,2, \ldots n\}} \min \left(\pi\left(x_{i}\right), 1\right) \quad \rho(\underline{x})=\max _{i \in\{1,2, \ldots n\}} \min \left(\pi\left(x_{i}\right), \mu_{i}\right)
$$

and for all other $x \in X$

$$
\rho(x)=\max _{i \in\{1,2, \ldots n\}} \min \left(\pi\left(x_{i}\right), 0\right)=0
$$

We have $\rho(\bar{x})=1$ because $\max _{i}\left(\pi\left(x_{i}\right)\right)=1$. Thus $\pi \sim[1 / \bar{x}, \rho(\underline{x}) / \underline{x}]$.

Now we turn to $A 2^{-}$. Suppose $\pi_{1} \geq \pi_{2}$. We show that $\pi_{1} \sim\left[1 / \bar{x}, \rho_{1}(\underline{x}) / \underline{x}\right)$ and $\pi_{2} \sim\left[1 / \bar{x}, \rho_{2}(\underline{x}) / \underline{x}\right]$ where $\rho_{r}(\underline{x}) r=1,2$ is calculated by equation $(37)$. Since $\pi_{1} \geq \pi_{2}$, and functions min, max are increasing on each argument, we have $\rho_{1}(\underline{x}) \geq \rho_{2}(\underline{x})$. By $B 2$, we have $\left[1 / \bar{x}, \rho_{2}(\underline{x}) / \underline{x}\right] \succeq\left[1 / \bar{x}, \rho_{1}(\underline{x}) / \underline{x}\right]$. From this, by transitivity we have $\pi_{2} \succeq \pi_{1}$.

Corollary 1 System $\mathcal{S}_{P}\left(\mathcal{S}_{O}\right)$ is a special case of $\mathcal{S}$ when each prize in $X$ has an equivalent canonical lottery in $\mathcal{C}^{-}\left(\mathcal{C}^{+}\right)$.

It is clear that $\mathcal{S}$ is more general than either $\mathcal{S}_{P}$ or $\mathcal{S}_{O}$ because $\mathcal{C}^{-}$and $\mathcal{C}^{+}$are just two proper subsets of $\mathcal{C}$.

This corollary also demonstrates the point made earlier that, in our view, there is no real need for separating decision making with possibility theory into the pessimistic or the optimistic modes. In our possibilistic decision theory, the "pessimistic behavior" is the result of assigning gain utilities to prizes whereas the "optimistic behavior" is the result of assigning loss utilities to prizes. In other words, there is one utility theory that works for all possibilistic decision makers. And the difference in observed behaviors can be explained by different utility assignments. Example 2 illustrates this fact. Case 1, involving gains only, corresponds to a pessimistic mode; case 2, with loss prizes, to an optimistic mode. However, case 3 involving both gains and losses could be handled in neither pessimistic nor optimistic framework.

Since binary utility is represented by two numbers whereas pessimistic and optimistic utility by one number each, a question that could be raised is whether coupling $Q U^{-}, Q U^{+}$into a lexicographic order would yield $P U$.

In [5], Dubois et al. consider a lexicographic aggregation of pessimistic and optimistic rankings in order to increase the discrimination power. Let us summarize their result by skipping nonessential technical details. The authors consider $\left(M A 0_{\top}^{g}-\right.$ page $\left.435,[5]\right)$ two preference relations $\succeq^{-}$and $\succeq^{+}$that satisfy $\mathcal{S}_{P}$ and $\mathcal{S}_{O}$ respectively. In addition, these preference relations are assumed to satisfy 3 conditions as follows.

(1) $\forall x, y \in X, x \succeq^{-} y$ iff $x \succeq^{+} y$

(2) $\forall \lambda, \mu \in V,[1 / \bar{x}, \lambda / \underline{x}] \succ^{-}[1 / \bar{x}, \mu / \underline{x}]$ iff $[\mu / \bar{x}, 1 / \underline{x}] \succ^{+}[\lambda / \bar{x}, 1 / \underline{x}]$

(3) The number of equivalence classes by indifference relations is the same as the number of prizes i.e., $\left|\Pi_{X} / \sim^{-}\right|=\left|\Pi_{X} / \sim^{+}\right|=|X|$ 
Given such $\succeq^{-}, \succeq^{+}$, a new preference relation $\succeq^{-+}$can be defined by lexicographic aggregation as

$$
\pi_{1} \succ^{-+} \pi_{2} \text { iff }\left(\pi_{1} \succ^{-} \pi_{2}\right) \vee\left(\pi_{1} \sim^{-} \pi_{2} \& \pi_{1} \succ^{+} \pi_{2}\right)
$$

Because of representation theorems for $\mathcal{S}_{P}$ and $\mathcal{S}_{O}$, one would expect that this $\succeq^{-+}$could be represented by a lexicographic aggregation of pessimistic and optimistic utilities $\left(Q U^{-}, Q U^{+}\right)$. Indeed, it is the case (theorem 3, page 435).

With the purpose of forming a more discriminant order, one can, in principle, aggregate any number of preference orders (not necessary a combination of a pessimistic and a optimistic) without regards to the existence of a consistent axiom system underlying the aggregated preference. The only condition necessary for this type of aggregation to make sense is the establishment of a priority order over the agents or the criteria. For the lexicographic aggregation of $\succeq^{-}$and $\succeq^{+}$, the inconsistency of underlying axiom systems $\mathcal{S}_{P}$ and $\mathcal{S}_{O}$ is a non-issue.

A lexicographic utility can be used, as Fishburn [8] has shown, to represent certain axiom systems, which do not have a convenient representation by a real value utility function. The study of binary utility in this paper falls in this category. Given that consideration, the syntactical similarity between the binary utility $(P U)$ and the aggregation of pessimistic and optimistic utilities should not blur the fact that they are designed for different situations. The former is to represent the preference, based on a single decision criterion (implicit in the total pre-order axiom), of a single (rational) decision maker. The latter is supposed to reflect a situation involving more than one agents (sources or decision criteria). Therefore, there is hardly a common ground to compare $\succeq_{P U}$ and $\succeq^{-+}$except on the discrimination power dimension.

In the formulation of $M A 0_{T}^{g}$, which enables a representation theorem, there is one condition that seriously impairs the purpose of increasing discrimination power. Condition (3) requires that the number of equivalence classes formed by each relation $\succeq^{-}, \succeq^{+}$is the same as the number of prizes $(|X|)$. So in combination, $\succeq^{-+}$can deliver $|X|^{2}$ equivalence classes at most. This upper bound is independent of the refinement degree (cardinality) of uncertainty scale. A preference induced by Wald's minimax rule has a similar property. In an extreme example, if $X=\{\bar{x}, \underline{x}\}, \succeq^{-+}$can separate $\Pi_{X}$ into four levels at most. In contrast, discrimination power of $\mathcal{S}$ correlates with the refinement degree of the uncertainty scale because the set of prototypes on which all lotteries are compared with is taken to be the set of canonical lotteries $(|\mathcal{C}|=2|V|-1)$ assuming only $|\mathcal{C}| \geq|X|$ (axiom B4). This property is shared by a preference relation represented by vNM linear utility. For example with $X=\{\bar{x}, \underline{x}\}$ and 10-level uncertainty scale $(|V|=10)$, a preference relation $\succeq$ satisfying $\mathcal{S}$ divides $\Pi_{X}$ into 19 equivalence classes.

The above arguments make clear that the reasoning based on binary utility should not be viewed as a combination of pessimistic and optimistic reasonings. 


\section{Summary and Conclusion}

In this paper we have proposed a new system of axioms for decision making with possibility theory. Our axiomatic system $(\mathcal{S})$ unifies and generalizes the pessimistic and optimistic systems of axioms $\left(\mathcal{S}_{P}, \mathcal{S}_{O}\right)$ previously proposed by Dubois et al. The unification is made by $(i)$ substituting two informational attitude axioms expressing pessimism and optimism (uncertainty aversion and uncertainty attraction) by the monotonicity axiom $B 2$ that could be intuitively justified in the spirit of "sure-thing" principle; (ii) generalization of continuity axioms. Pessimistic and optimistic systems are proper special cases of the new system. Our system handles lotteries involving both gains and losses whereas the pessimistic system works for lotteries involving gains only and the optimistic system - those with losses only. Conceptually, in our proposal, uncertainty attitude is implicit in the individual utility functions rather than explicit as an axiom.

We also prove a representation theorem for the unified axiom system. Any preference relation satisfying the axiom system can be represented by a utility function that maps lotteries into an linearly ordered binary utility scale where each utility level is a pair of numbers. The utility function is a composition of $\max$, min operations that have been generalized in a natural way to work with pairs. Structurally, it is similar to the composition of the classic expected utility expression where in place of addition and multiplication, max and min are used respectively.

It is well known that probability theory interprets negation operation in a strictly complementary sense i.e., $p(\neg A)=1-p(A)$. This fact makes it sufficient to represent the chance (or belief in Savage's personalistic view) of an event by one number - its probability. Unlike probability theory, possibility theory as well as other non-probabilistic uncertainty calculi e.g., Dempster-Shafer belief function theory $[15,16]$ or interval-valued probability $[12,19]$ describe uncertainty of an event by two numbers. They are possibility and necessity degrees in possibility theory; plausibility and belief in DS theory; upper and lower probabilities in interval-valued probability theory. It is also well known that the heart of decision making under uncertainty is the trade-offs between uncertainty and consequences. In order to enable the trade-offs, utility and uncertainty must be "comparable." For these reasons, we speculate that a binary utility scale is, perhaps, the "correct" answer to a binary representation of uncertainty. 


\section{References}

[1] Brafman, R. I., And Tennenholtz, M. An axiomatic treatment of three qualitative decision criteria. Journal of the Association of Computing Machinary 47, 3 (2000), 452-483.

[2] Doyle, J., And Thomason, R. Background to qualitative decision theory. AI Magazine 20, 2 (1999), 55-68.

[3] Dubois, D., Fodor, J. C., Prade, H., and Roubens, M. Aggregation of decomposable measures with application to utility theory. Theory and Decision 41 (1996), 59-95.

[4] Dubois, D., Godo, L., Prade, H., and Zapico, A. On the possibilistic decision model: from decision under uncertainty to case-based decision. International Journal of Uncertainty, Fuzziness and Knowledge- based Systems 7, 6 (1999), 631-670.

[5] Dubois, D., Godo, L., Prade, H., and Zapico, A. Advances in qualitative decision theory: Refined rankings. In Lecture Notes in Artificial Intelligence (11 2000), M. C. Monard and J. S. Sichman, Eds., vol. 1952, Springer, pp. $427-436$.

[6] Dubois, D., Nguyen, T. H., And Prade, H. Possibility theory, probability and fuzzy sets. In Handbook of Fuzzy Sets Series, D. Dubois and H. Prade, Eds. Kluwer Academic, Boston, 2000, pp. 344-438.

[7] Dubois, D., Prade, H., and Sabbadin, R. Decision-theoretic foundation of qualitative possibility theory. European Journal of Operational Research 128 (2001), 478-495.

[8] Fishburn, P. C. Lexicographic orders, utilities and decision rules: A survey. Management Scicence 20, 11 (1974), 1442-1471.

[9] Giang, P. H., And Shenoy, P. P. A qualitative linear utility theory for Spohn's theory of epistemic beliefs. In Uncertainty in Artificial Intelligence: Proceedings of the Sixteenth Conference (UAI-2000) (San Francisco, CA, 2000), C. Boutilier and M. Goldszmidt, Eds., Morgan Kaufmann, pp. 220229.

[10] Giang, P. H., And Shenoy, P. P. A comparison of axiomatic approaches to qualitative decision making using possibility theory. In Uncertainty in Artificial Intelligence: Proceedings of the Seventeenth Conference (UAI2001) (San Francisco, CA, 2001), J. Breese and D. Koller, Eds., Morgan Kaufmann, pp. 162-170.

[11] Giang, P. H., And Shenoy, P. P. Statistical decisions using likelihood information without prior probabilities. In Uncertainty in Artificial Intelligence: Proceedings of the Eighteenth Conference (UAI-2002) (San Francisco, CA, 2002), A. Darwiche and N. Friedman, Eds., Morgan Kaufmann, pp. $170-178$. 
[12] Levi, I. On indeterminate probabilities. Journal of Philosophy 71 (1974), 391-418.

[13] SARin, R., AND WAKker, P. A simple axiomatization of nonadditive expected utility. Econometrica 60, 6 (1992), 1255-1272.

[14] Schmeidler, D. Subjective probability and expected utility without additivity. Econometrica 5\%, 3 (1989), 571-587.

[15] Shafer, G. A Mathematical Theory of Evidence. Princeton University Press, Princeton, NJ, 1976.

[16] Smets, P. The transferable belief model for quantified belief representation. In Handbook of Defeasible Reasoning and Uncetainty Management system, D. M. Gabbay and P. Smets, Eds., vol. 1. Kluwer, Doordrecht, 1998, pp. 267-301.

[17] Sporn, W. Ordinal conditional function: A dynamic theory of epistemic states. In Causation in Decision, Belief Change and Statistics (1988), L. Harper and B. Skyrms, Eds., Kluwer Academic, pp. 105-134.

[18] von Neumann, J., and Morgenstern, O. Theory of Games and Economic Behavior, 3 ed. Princeton University Press, Princeton, NJ, 1953. 1st edition 1944.

[19] Walley, P. Statistical Reasoning with Imprecise Probabilities. Chapman and Hall, London, New York, 1991.

[20] Zadeh, L. Fuzzy set as a basis for a theory of possibility. Fuzzy Sets and Systems 1 (1978), 3-28. 